\title{
Study on friction behavior at the interface between prosthetic socket and liner
}

\author{
JiNGYANG XIE ${ }^{1}$, XIDONG LIU ${ }^{2}$, JiANHUA TANG ${ }^{1}, \mathrm{XI} \mathrm{LI}^{3}$, WeI LI ${ }^{1}$ * \\ ${ }^{1}$ Tribology Research Institution, Key Laboratory for Advanced Technology of Materials of Ministry of Education, \\ Southwest Jiaotong University, Chengdu, People's Republic of China. \\ ${ }^{2}$ Affiliated Sichuan Provincial Rehabilitation Hospital of Chengdu University of TCM, Chengdu, People's Republic of China. \\ ${ }^{3}$ China Disabled Assistive Devices Center, Beijing, People's Republic of China.
}

\begin{abstract}
Purpose: The friction characteristics at the interface between prosthetic socket and liner have an important influence on the walking function and wearing comfort of amputees. The frictional behavior at the prosthetic socket/liner interface can provide theoretical guidance for the design and selection of prosthetic materials. So it is of great significance to study the friction behavior at prosthetic socket/liner interface. Methods: The surface roughnesses of the prosthetic socket and liner materials were measured by a laser confocal microscope. The frictional behavior at the prosthetic socket/liner interface was studied on a UMT TriboLab Tribometer by simulating the reciprocating sliding contact mode. An infrared camera was used to take thermal images and then calculated the temperature increase at the socket/liner interface. Results: The coefficient of friction of the silicon rubber fabric are significantly smaller than that of the foam liner materials. The frictional energy dissipation at the liner/acrylic socket interface is the smallest, while it is greater for 3D-printed socket materials. Meanwhile, the temperature increase has a positive correlation to the coefficient of friction and frictional energy dissipation. Conclusions: The three kinds of 3D-printed materials with high surface roughness have higher interface coefficient of friction and energy dissipation than acrylic material. The stiffness and energy consumption play an important role in the interface friction characteristics of the prosthetic liner materials. The appropriate coefficient of friction at the surface between prosthetic socket and liner is essential. A type of the reinforcement fiber has influence on the friction behavior of the 3D-printed reinforced nylon.
\end{abstract}

Key words: prosthetic materials, interface friction, energy dissipation, stiffness

\section{Introduction}

At present, the number of amputees is increasing all over the world and wearing prosthesis is a proper way for amputees to restore the walking function and reintegrate into society. It is also a primary choice for rehabilitation treatment of amputees worldwide [17], [19]. The design of the prosthetic socket and liner should not only assist the patient to walk properly, but also meets the patients' wearable comfort requirements [9]. As a result, meeting these requirements involves not only the structural design of the prosthetic socket and liner, but also the inherent mechanics and surface properties of the materials.
The simplified force model of the lower limb prosthetic system is shown in Fig. 1. The interfacial friction between the skin, socket and liner plays a significant role for the design and use of prostheses. At present, researchers mainly studied the prosthetic system from finite element simulation (FEM), materials of prostheses and the friction between prosthetic materials and skin etc. Zhang et al. [22], [23] analyzed the friction between the lower leg residual limb and the prosthetic socket using geometric models, finite element models and clinical stress tests, concluding that friction plays an important role in supporting the body weight. Besides, the suspension of the prosthesis prevents shedding during the walking period. Lee et al. [11] proposed a new practical approach in modeling of the

\footnotetext{
* Corresponding author: Wei Li, Tribology Research Institute, Key Laboratory for Advanced Technology of Materials of Ministry of Education, Southwest Jiaotong University, Chengdu 610031, China. E-mail: liweijiani@home.swjtu.edu.cn

Received: November 10th, 2020

Accepted for publication: January 5th, 2021
} 
contact interface with consideration of the friction/slip conditions and pre-stresses applied on the limb within a rectified socket. Zhang et al. [21] predicted the pressure and shear stress distribution at the limb/socket interface by FEM and found that the coefficient of friction was a sensitive parameter in determining the interface pressures, shear stresses and slip. Feng et al. [6] designed three types of bionic texture surfaces to investigate reciprocating friction characteristics. The results showed that the texture had a significant effect on the surface characteristics and the friction characteristics of the skin. Kong et al. [10] studied the tribological characteristics at the interface between different prosthetic sockets and skin and found that the order of frictional coefficient between socket materials and skin is: silicone rubber-acrylic resin-polyethylene sheet-cotton liner. Li et al. [13] found that the tribological behavior, mechanical stimulation and comfort of the skin were closely related to the weaving parameters, surface characteristics and material components of the liner fabric. At present, it is generally believed that the mechanical analysis of the interface between the residual limb and the prosthetic socket is the basis for the design and fabrication of the prosthetic socket. Therefore, for more accurate analysis, scholars have studied the 3D reconstruction and mechanical analysis of residual limb and prostheses [2], [3], [7], [18], [25].

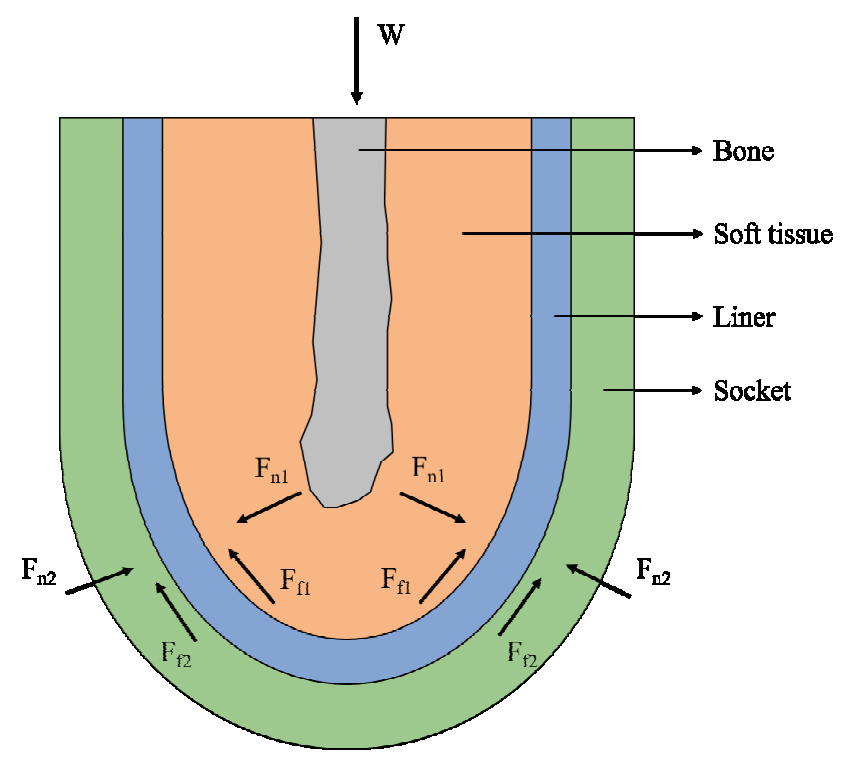

Fig. 1. The simplified model of prostheses system.

$W$ represents body weight; $F_{n 1}$ and $F_{f 1}$ represent normal force and frictional force exerted by the soft tissue on the liner, respectively; $F_{n 2}$ and $F_{f 2}$ represent normal force and frictional force exerted by the socket on the liner, respectively

In summary, the previous studies mainly focused on the prosthetic socket/skin and prosthetic liner/skin interface, and only few reports on the friction behavior at the prosthetic socket/liner interface. For the foam liner, it is attached to the prosthetic socket by friction. When the patient's residual limb is in the swing phase, the static friction and pre-tension at the prosthetic socket/skin interface plays a significant role in preventing the prosthesis from falling off. Under the same pre-tension force, the relative sliding is likely to occur due to small coefficient of friction. It may cause the weak stability of the prosthetic system and a poor posture during walking period.

Due to the above-mentioned reasons, the friction behavior at the prosthetic socket/liner interface was studied by simulating the reciprocating sliding contact mode between the prosthetic socket and liner on a UMT TriboLab Tribometer in this paper. The prosthetic socket materials were 3D-printed and handmade, and the prosthetic liner materials were foam and silicone rubber. The results can provide theoretical guidance for the design and selection of prosthetic materials.

\section{Materials and methods}

\subsection{Prosthetic materials}

Four kinds of socket materials and three kinds of liner materials were selected in this study. The materials of the three 3D-printed socket ball samples (Figs. 2a-c) were FS 3300PA nylon, FS 3400GF glass fiber-reinforced nylon and FS 3400CF carbon fiber-reinforced nylon printed by SLS (Selective Laser Sintering) method in HT403P 3D printer (Farsoon, China). The acrylic material (Fig. 2d, handmade by Affiliated Sichuan Provincial Rehabilitation Hospital of Chengdu University of TCM) was mainly made of acrylic, sponge, nylon and carbon fiber. The diameter of all the sockets' hemisphere was $20 \mathrm{~mm}$. In Figs. 2e-g, three kinds of prosthetic liner materials, including two types of foam liner and one type of silicone rubber liner are shown. The two types of foam liner were Ottobock liner plates $617 \mathrm{~S} 26=\mathrm{H} 5$ (Fig. 2e, Ottobock, Germany) and Jingbo liner plates (Fig. 2f, Jingbo, China), with thicknesses of $5.0 \mathrm{~mm}$ and $5.5 \mathrm{~mm}$, respectively. The silicon rubber liner was Össur ASPIRE prosthetic liner (Fig. 2g, silicon rubber, Össur, Iceland). It has two layers: a silicone rubber layer and a fabric layer. Each prosthetic liner material was cut into a rectangular sample with size of $42 \times 30 \mathrm{~mm}$ and fixed on a stainless steel plate with strong glue. Besides, the surface roughnesses of the prosthetic socket and liner 
(a)

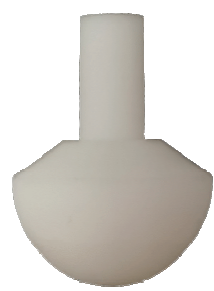

(e)
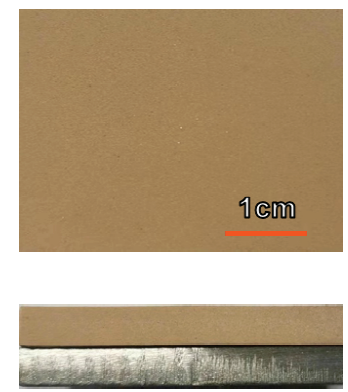

(f)

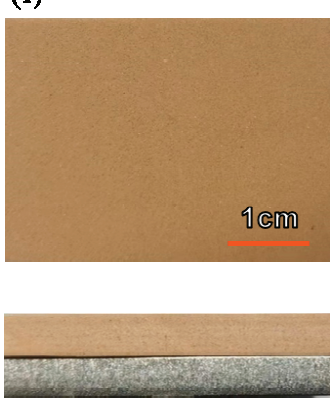

(c)
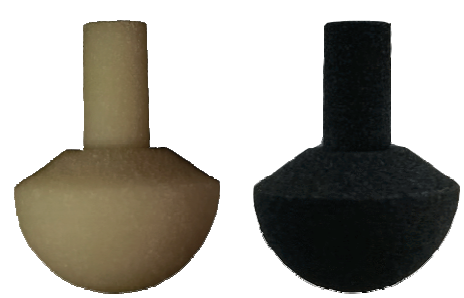

(d)

(g)

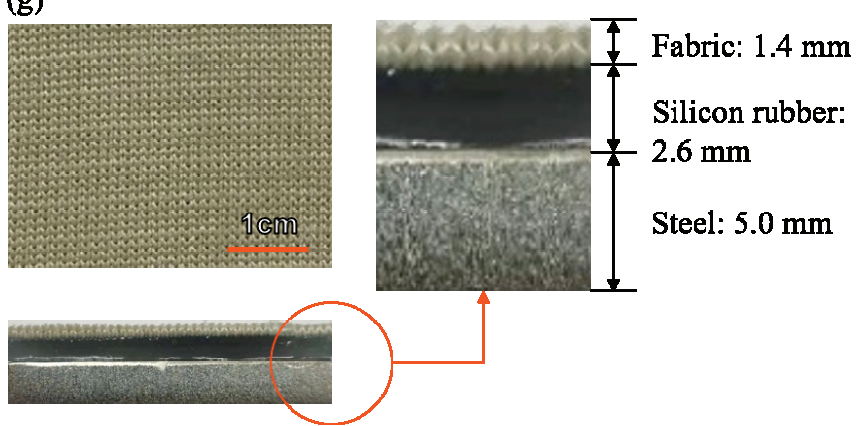

Fig. 2. The socket ball samples and the exterior of prosthetic liner materials: (a) nylon, (b) glass fiber nylon, (c) carbon fiber nylon, (d) handmade acrylic, (e) Ottobock foam liner, (f) Jingbo foam liner, $(\mathrm{g})$ silicon rubber with two layers: fabric $(1.4 \mathrm{~mm})$ and silicon rubber $(2.6 \mathrm{~mm})$

materials were measured by a laser confocal microscope (KEYENCE VK-X1000, Japan). Each material was tested for five times at five random positions.

\subsection{Friction test}

The friction test was performed under a UMT TriboLab Tribometer (Fig. 3, Bruker, USA). The reciprocating sliding mode was used to simulate the interface friction between different types of prosthetic socket and liner materials. According to the mechanical analysis by Zhang et al. [23], the normal loads in this study were $0.8 \mathrm{~N}$ and $8.0 \mathrm{~N}$, which referred to about $41 \mathrm{kPa}$ and $89 \mathrm{kPa}$ of the contact pressure, respectively. The reciprocating displacement $D$ was $\pm 5 \mathrm{~mm}$, the frequency $f$ was $0.5 \mathrm{~Hz}$, which simulated step frequency of amputee during walking period [11], [23]. Repeated tests were carried out three times for each condition. The number of reciprocating cycles was 150 , corresponding to $5 \mathrm{~min}$ walking time. All the tests were performed at a temperature of $20 \pm 2{ }^{\circ} \mathrm{C}$ and a relative humidity of 60 $\pm 2 \%$. The infrared thermal camera (FLIR E40, USA) was used to take 5 thermal images of the friction area during each test. And then the temperature increase can be calculated. (a)

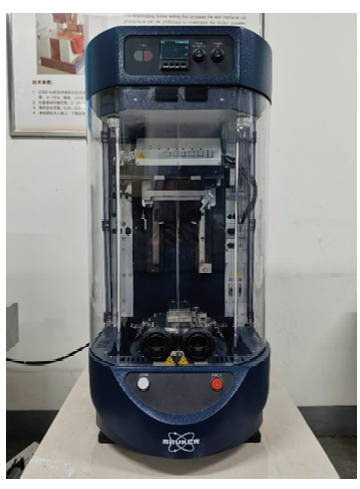

(b)

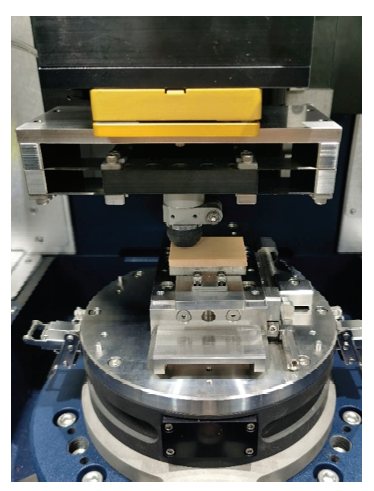

(c)

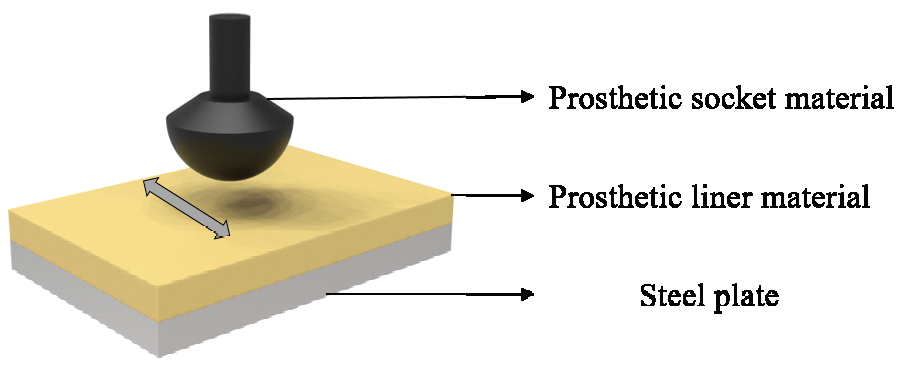

Fig. 3. The setup of the friction experiment: (a) UMT TriboLab Tribometer,

(b) reciprocating on UMT TriboLab Tribometer, (c) the schematic diagram of the friction test 


\subsection{Compression test of prosthetic liner materials}

The compression performance of the prosthetic liner materials was also tested on the UMT TriboLab Tribometer. The linear loading-unloading motion mode was used to test the stiffness of different prosthetic liner materials under different loads. The loading and unloading curves in a loading-unloading cycle were not coincidence, and the area enclosed by the curves was the internal energy consumption of the liner materials [15].

The ball probe for the compression test was a stainless steel ball with a diameter of $15 \mathrm{~mm}$. The prosthetic samples were the three kinds of liner materials, and they were the same as the samples in friction test. The normal loads were $0.5 \mathrm{~N}, 1.0 \mathrm{~N}, 1.5 \mathrm{~N}$ and $2.0 \mathrm{~N}$. The test process was as follows: step 1 - loading to $0.2 \mathrm{~N}$ and holding for $10 \mathrm{~s}$; step 2 - linear loading (60 s) to the required load; step 3 - linear unloading (60 s) to $0.2 \mathrm{~N}$; and step 4 - holding for $10 \mathrm{~s}$ under $0.2 \mathrm{~N}$. Each compression test repeated step 2 to step 4 four times, and the last loading-unloading cycle data was used for data analysis. For each kind of prosthetic liner material, three replicate tests were performed under the same test conditions.

\subsection{Statistical analysis}

Independent $t$-test was used to compare the coefficient of friction and loading-unloading data under different circumstances. $F$-test (analysis of variance) was used to determine the dramatically difference among repeated three tests under the same conditions. The level of statistical significance was set to $P<0.05$.

\section{Results}

\subsection{Surface properties of prosthetic socket and liner materials}

In Figure 4, the 2D and 3D surface topographies of the socket and liner materials measured by LCSM

(c)

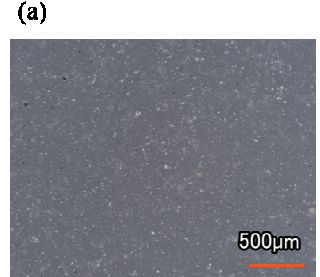

(b)
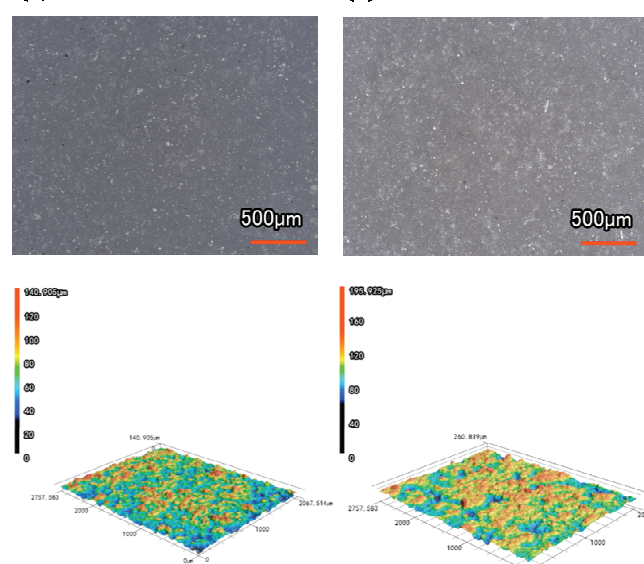

(e)
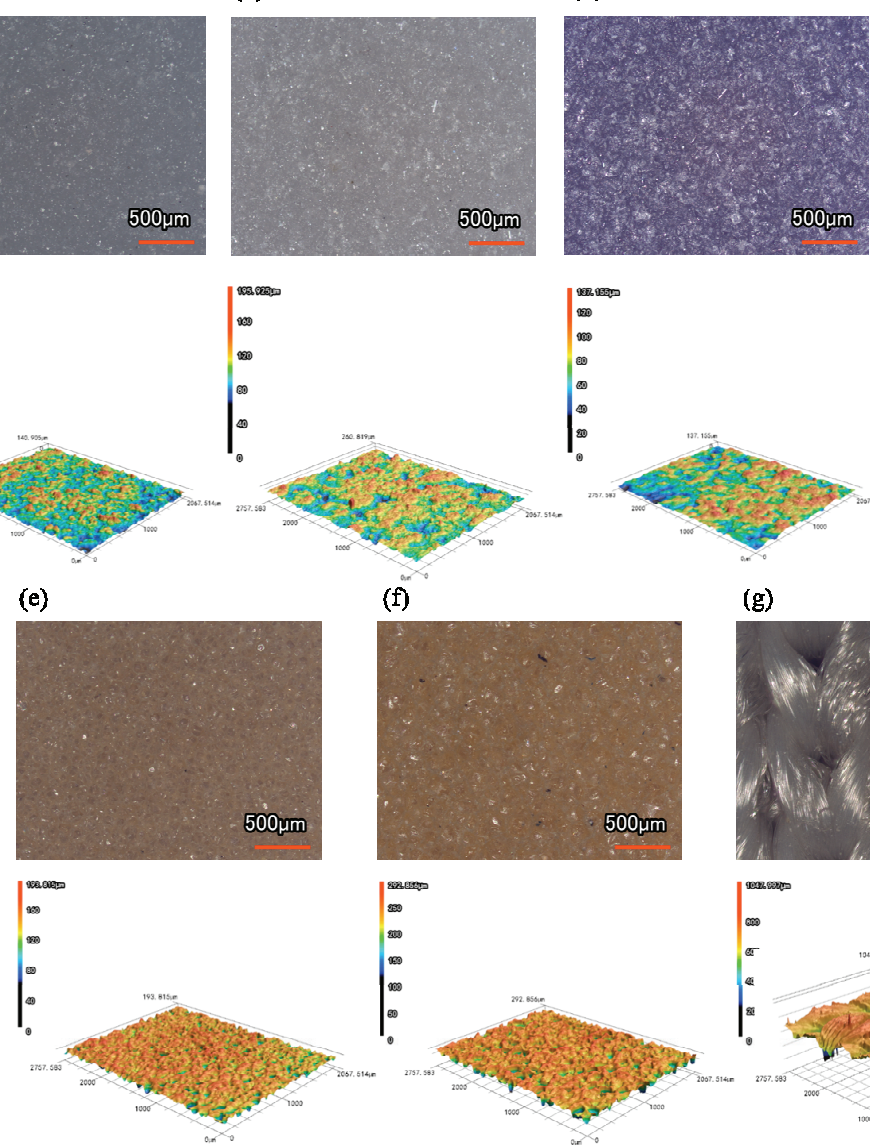

(d)
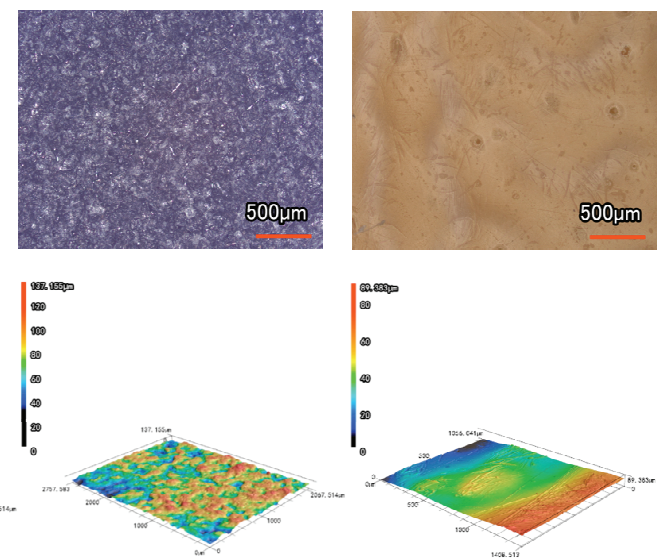

(g)
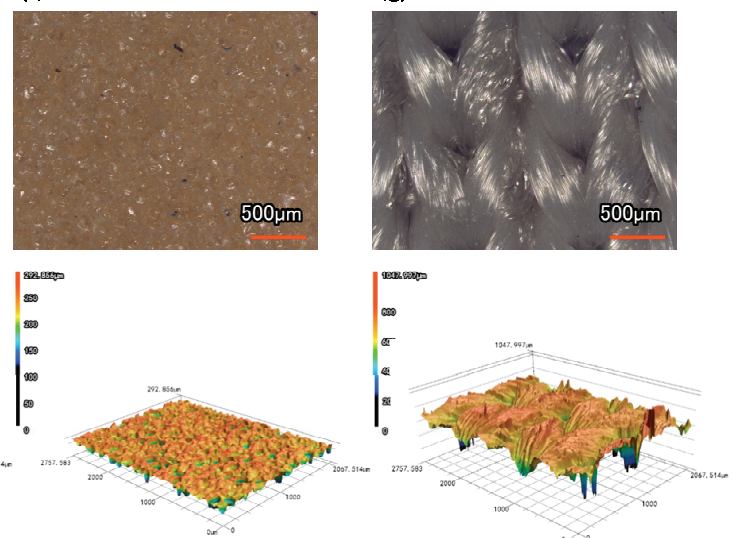

Fig. 4. The surface topographies of socket and liner materials: (a) nylon, (b) glass fiber reinforced nylon, (c) carbon fiber reinforced nylon, (d) acrylic, (e) Ottobock foam liner, (f) Jingbo foam liner, (g) fabric on Össur silicon rubber liner 
(Confocal Laser Scanning Microscope, KEYENCE VK-X1000, Japan) are shown. In Table 1, the surface roughnesses of the prosthetic materials are listed. Regarding the socket materials, the handmade acrylic material is comparatively smooth with the lowest surface roughness. For the 3D-printed socket materials, the glass fiber reinforced nylon has the highest surface roughness, while the other two 3D-printed materials are close to each other. For the liner materials, the surface roughness of the Ottobock foam liner is slightly lower than that of Jingbo foam liner, and the surface roughness of the fiber fabric on silicon rubber liner is the highest.
In Figure 5, the typical surface topographies of the liner materials after friction with the nylon prosthetic socket under normal load of $8.0 \mathrm{~N}$ taken with camera are shown. As can be seen, the scratch of Jingbo foam liner is severer. Meanwhile, in Fig. 6, the surface roughnesses of different socket and liner materials before and after friction test are summarized. It is evident that the surface roughnesses of foam liner materials have an increasing trend after friction with different socket materials. However, for the same socket material, the roughness increase of Jingbo foam liner is greater than that of Ottobock foam liner. In addition, the surface roughness of the

Table 1. The surface roughnesses of prosthetic sockets and liners

\begin{tabular}{|c|c|c|c|c|}
\hline Prosthetic socket & Nylon & Glass fiber nylon & Carbon fiber nylon & Acrylic \\
\hline Roughness $[\mu \mathrm{m}]$ & $13.5 \pm 0.6$ & $15.3 \pm 0.9$ & $13.3 \pm 0.4$ & $10.0 \pm 0.8$ \\
\hline Prosthetic liner & Ottobock foam & Jingbo foam & Fabric on Össur silicon rubber & Össur Silicon rubber \\
\hline Roughness $[\mu \mathrm{m}]$ & $17.0 \pm 0.3$ & $19.3 \pm 0.5$ & $53.3 \pm 3.1$ & - \\
\hline
\end{tabular}

(a)

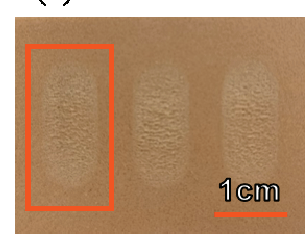

(e)

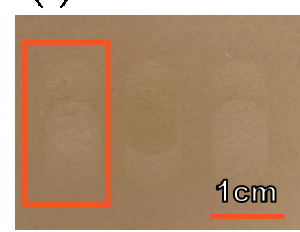

(b)

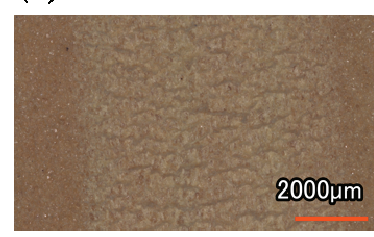

(f)

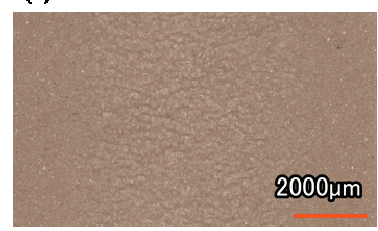

(c)

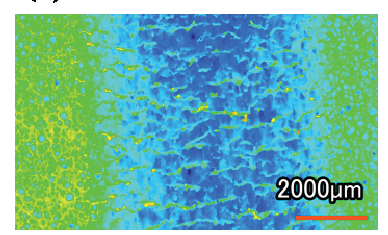

(g)

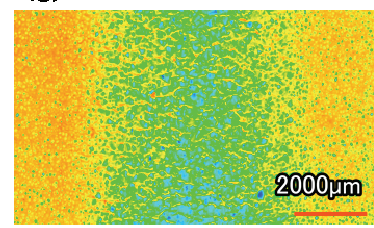

(d)

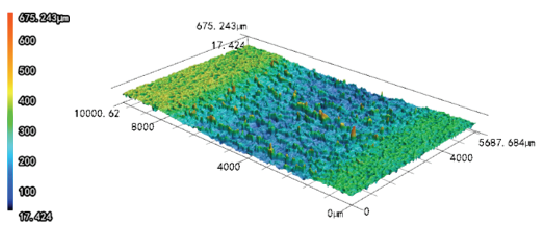

(b)

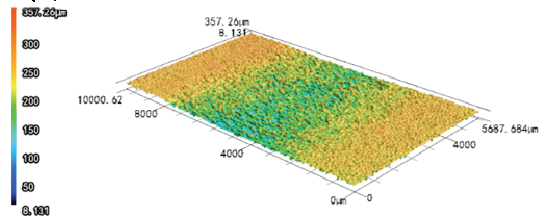

Fig. 5. The typical surface topographies of different liner materials after friction with nylon prosthetic socket under normal load of $8.0 \mathrm{~N}$ : (a), (b) the scratches of Jingbo foam liner, (c) 2D scratch of Jingbo foam liner, (d) 3D scratch of Jingbo foam liner,

(e), (f) the scratches of Ottobock foam liner by Camera, (g) 2D scratch of Ottobock foam liner, (h) 3D scratch of Ottobock foam liner

Fig. 6. The surface roughness of different liner materials before and after friction with different socket materials under normal load of $8.0 \mathrm{~N}$

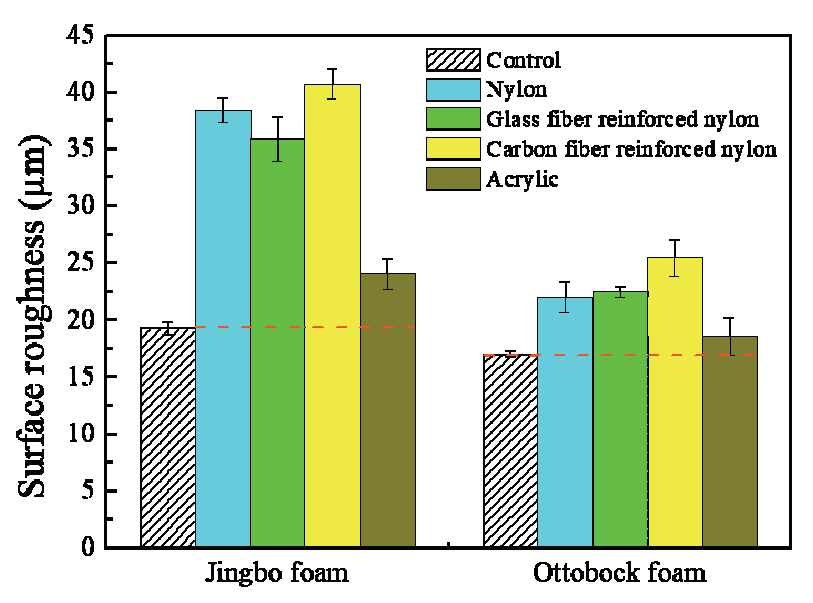

Prosthetic liner material 
two types of foam liner materials increases significantly after friction with the 3D-printed socket materials, while the roughness increase is comparatively smaller after friction with the acrylic material. Among the three kinds of 3D-printed socket materials, the surface roughnesses of the foam liners have the largest increase after friction with the carbon fiber reinforced nylon material.

\subsection{Friction characteristics under different normal loads}

\subsubsection{Coefficient of friction}

In Figure 7, the average coefficient of friction (COF) at different socket/liner interfaces under normal loads of $0.8 \mathrm{~N}$ and $8.0 \mathrm{~N}$ is shown. It is evident that the coefficient of friction of the silicon rubber fabric is considerably smaller than that of the foam liner materials. The overall coefficient of friction of the Ottobock foam liner is slightly smaller than that of the Jingbo foam liner, but the coefficient of friction of these two liners are not much different $(P<0.05)$. For the different socket materials, the coefficient of friction at the liner and the acrylic material interface is the smallest, while the coefficient of friction at the liner and the 3D-printed socket materials are comparatively larger. Moreover, the differencess among the coefficient of friction of the three 3D-printed socket materials are not significant $(P>0.05)$. While the normal load increased from $0.8 \mathrm{~N}$ to $8.0 \mathrm{~N}$, the coefficient of friction at the socket and liner interface increased slightly.

\subsubsection{Frictional energy dissipation analysis}

In Figure 8a, the typical curves of coefficient of friction between nylon socket material and three kinds of liner materials under normal load of $8.0 \mathrm{~N}$, and Figs. $8 \mathrm{~b}-\mathrm{d}$ are the related friction-displacement $(F-D)$ curves are shown. As can be seen, $F-D$ curves of the liner materials all present a parallelogram shape, indicating that the reciprocating friction movement of the interface between socket/liner materials is relative sliding. According to the method applied in our previous work [8], [12], [14], the three $F-D$ curves in Figs. 8b-d are integrated to calculate the energy dissipation in one reciprocating friction cycle.

In addition, in Figs. 8e-f, the average energy dissipation of one random cycle under normal loads of $0.8 \mathrm{~N}$ and $8.0 \mathrm{~N}$ are shown, respectively. Under different normal loads, the frictional energy dissipation at different socket/liner interfaces is different. Under the same normal load, the frictional energy dissipation at the different socket/liner interfaces also has a positive correlation to its coefficient of friction. By contrast, the frictional energy dissipation at the interfaces between silicon rubber fabric and the socket materials all significantly smaller than that of the foam liner materials. The overall frictional energy dissipation of the Ottobock foam is slightly smaller than that of the Jingbo foam $(P>0.05)$. In addition, for the different socket materials, the frictional energy (a)

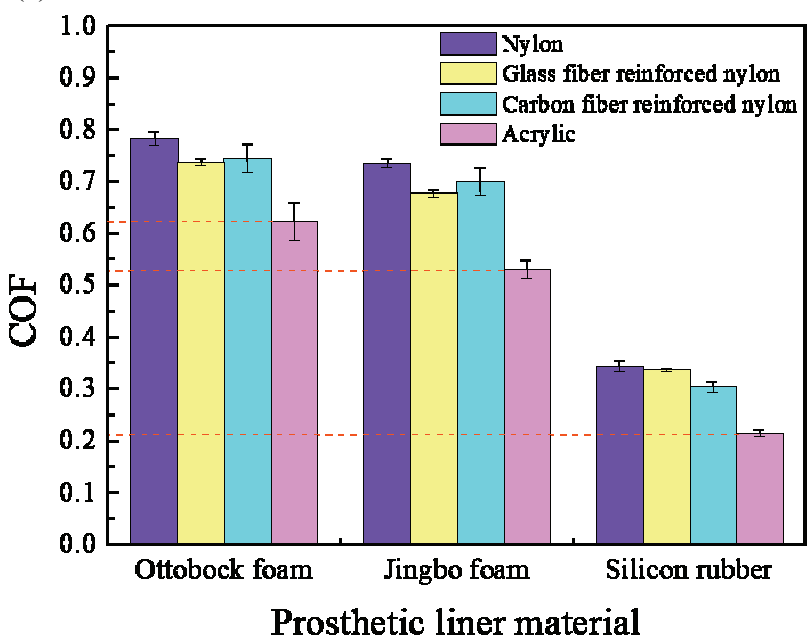

(b)

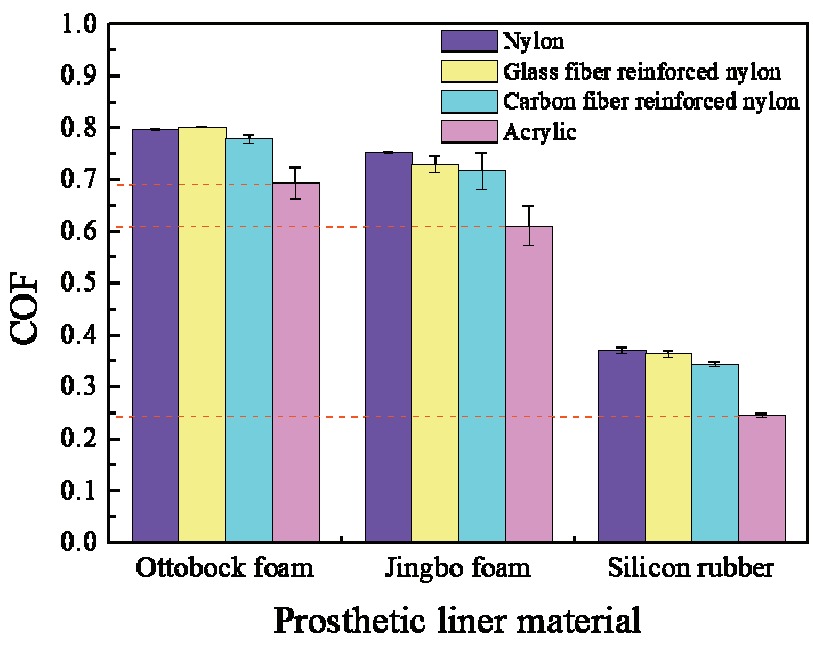

Fig. 7. The mean COF at different socket/liner interfaces: (a) under normal load of $0.8 \mathrm{~N}$, (b) under normal load of $8.0 \mathrm{~N}$ 
(a)

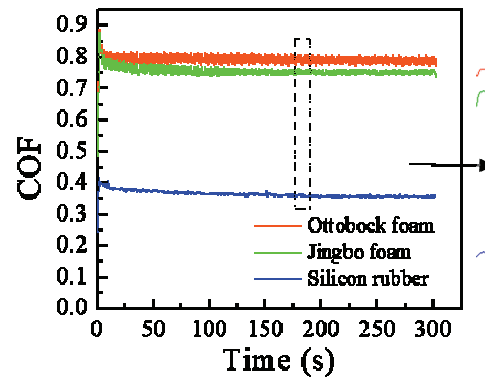

(c)

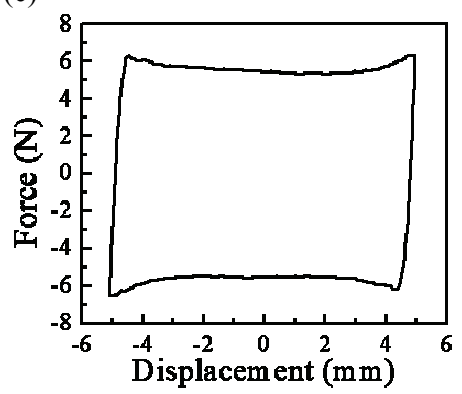

(e)

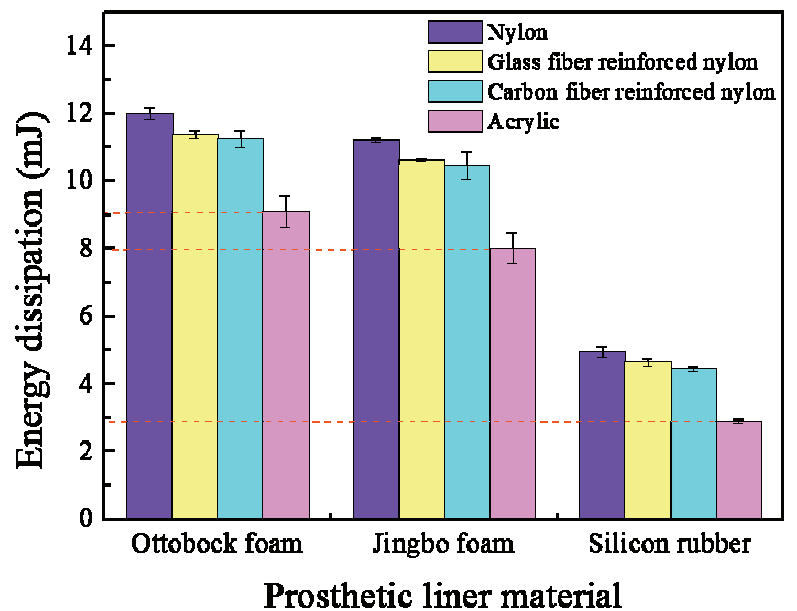

(b)

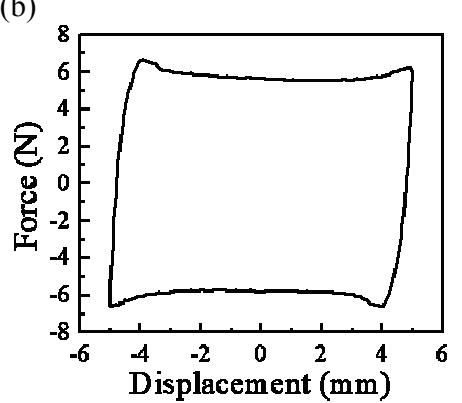

(d)

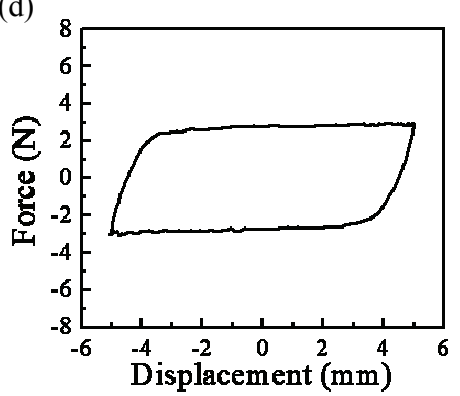

(f)

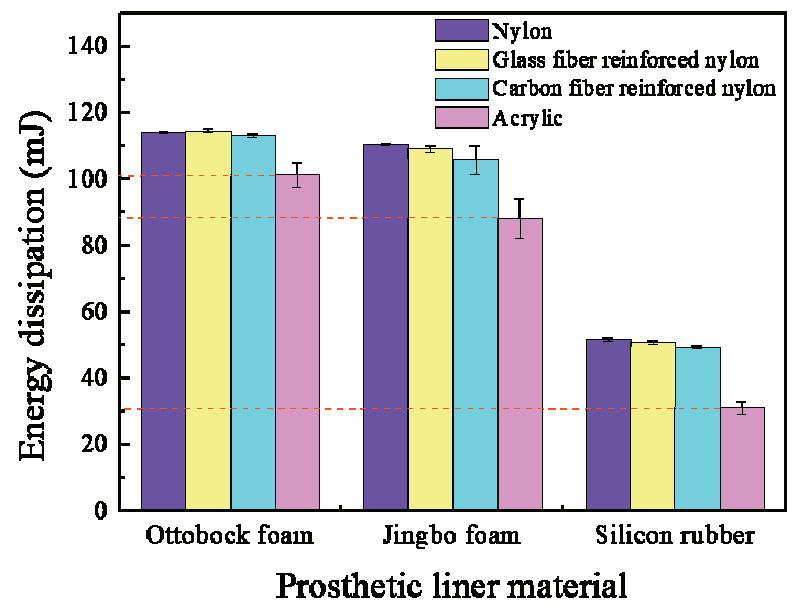

Fig. 8. The mean COF, typical $F-D$ curves in one reciprocating friction cycle between nylon socket material and different liner materials under normal load of $8.0 \mathrm{~N}$ and the frictional energy dissipation of different socket/liner material interfaces: (a) the COF between nylon material and different liner materials, (b) $F-D$ curve of nylon and Ottobock foam liner, (c) $F-D$ curve of nylon and Jingbo foam liner, (d) $F-D$ curve of nylon and fabric on silicon rubber liner, (e) energy dissipation under normal load of $0.8 \mathrm{~N}$, (f) energy dissipation under normal load of $8.0 \mathrm{~N}$

dissipation at the liner/acrylic socket interface is the smallest, while it is greater for 3D-printed socket materials $(P>0.05)$.

\subsubsection{Temperature increase at the socket/liner interface}

As shown in Figs. 9a-c, the typical temperature images of the friction area are taken with an infrared thermal camera (FLIR E40, USA). Figures 9d-e are the statistical results of the temperature increase at different socket/liner interfaces. As can be seen, the temperature value under normal load of $8.0 \mathrm{~N}$ is dramatically higher than that under normal load of $0.8 \mathrm{~N}$. Under the same load, the temperature increase of the fabric on silicon rubber liner and the four socket sample interfaces is significantly less than that of the foam liners. For the foam liners, the temperature increase of the Ottobock foam is slightly smaller than that of the Jingbo foam. The above results have a positive correlation to the $\mathrm{COF}$ and frictional energy dissipation. 
(a)

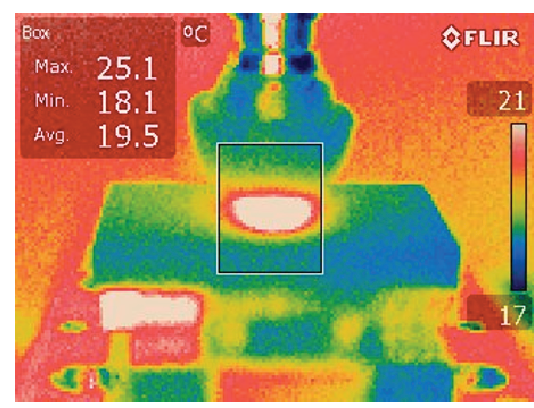

(d)

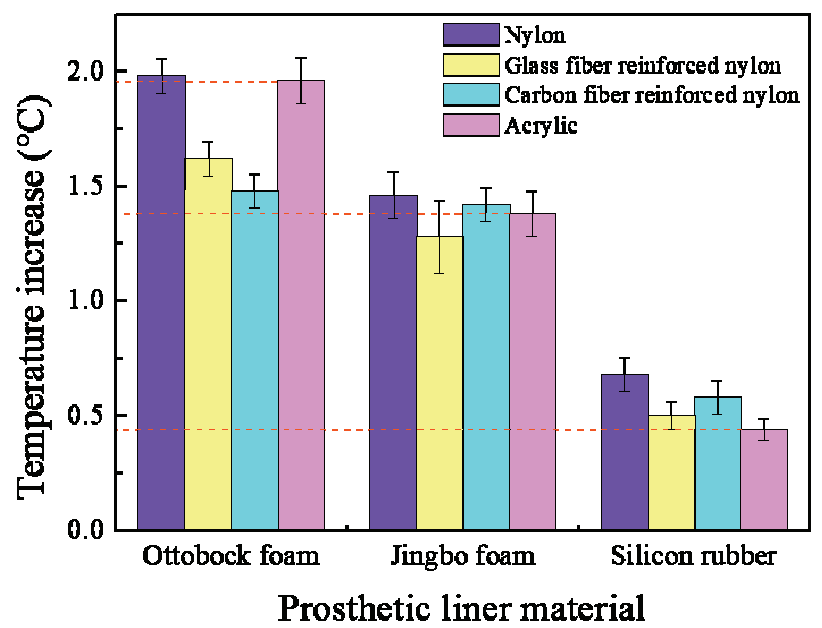

(b)

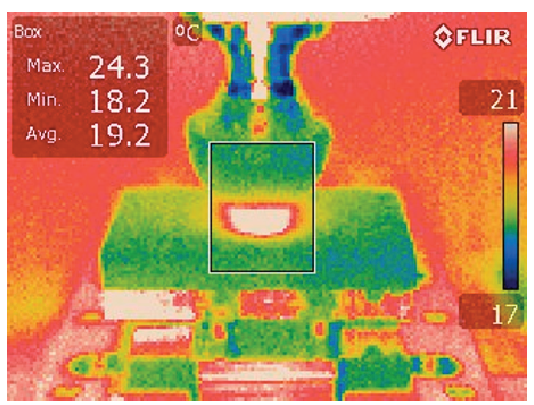

(e) (c)
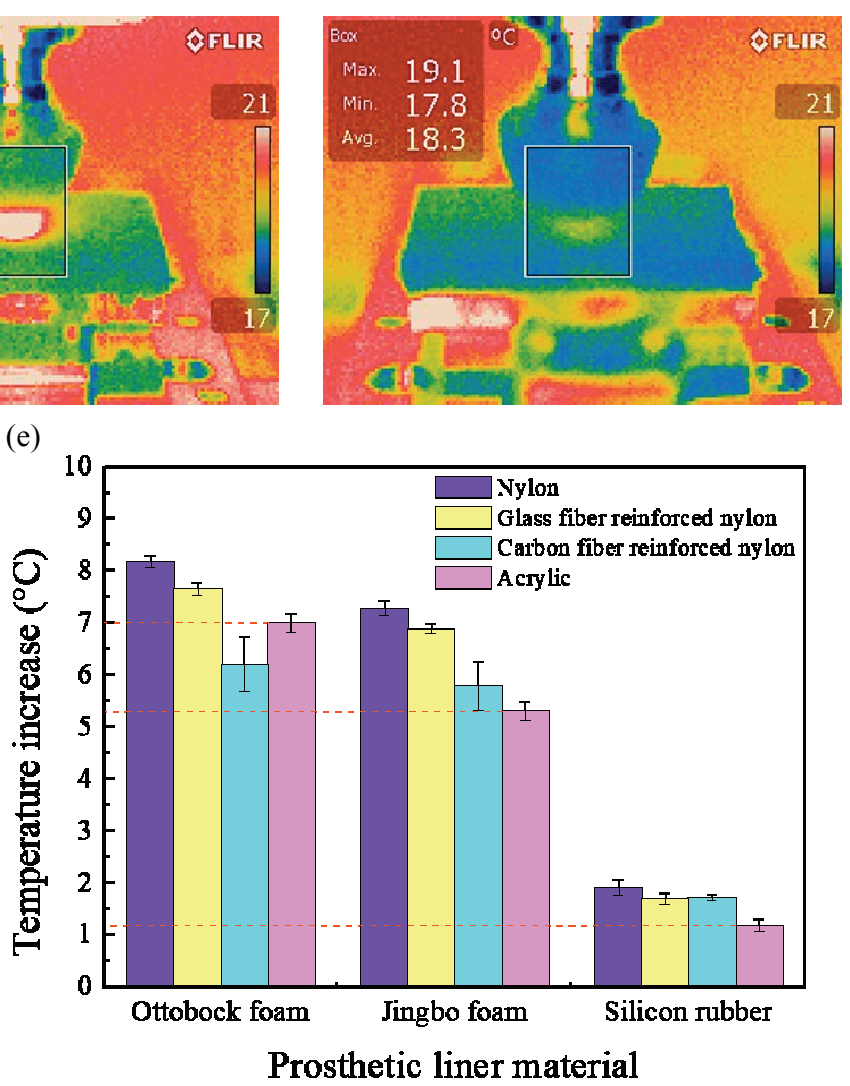

Fig. 9. The typical temperature images and temperature increase of different liner/socket interfaces: (a) acrylic/Ottobock foam liner, (b) acrylic/Jingbo foam liner, (c) acrylic/fabric on silicon rubber liner, (d) temperature increase under normal load of $0.8 \mathrm{~N}$, (e) temperature increase under normal load of $8.0 \mathrm{~N}$

\subsection{Stiffness of prosthetic liner materials}

Stiffness is the ability of a material or structure to resist elastic deformation when subjected to external force [16]. The typical loading-unloading curves of the three liner materials under different compressive loads $(0.5 \mathrm{~N}, 1.0 \mathrm{~N}, 1.5 \mathrm{~N}$ and $2.0 \mathrm{~N})$ are shown in Figs. $10 \mathrm{a}-\mathrm{d}$, and the slope of the curve is the stiffness of the material. Because the liners are viscoelastic materials, the loading and unloading curves are not overlap, and the area enclosed by these two curves is the internal energy consumption.

The stiffness of the liner materials is shown in Fig. 10e. As can be seen, the stiffness of the two foam materials is higher than that of the silicone rubber. With the increase of compressive load, the stiffness of all the liner materials increase gradually. In Figure 10f, the energy consumption of different liner materials in one loading-unloading cycle under different compressive loads is shown. Under the same compression load, the energy consumption between the Ottobock and
Jingbo foam is almost equal, while the energy consumption of silicon rubber is considerably greater than that of the two kinds of foam liner materials. In addition, the energy consumption of silicon rubber increased dramatically with the compressive load, which is much larger than that of the foam liner materials.

\section{Discussion}

The interface friction characteristics at the interface between the socket and liner have an important influence on the walking function and wearing comfort for amputees. In this paper, the friction properties at the prosthetic socket and liner interface were studied.

The coefficient of friction and energy dissipation between the socket and liner materials are directly related to the surface roughness and stiffness of the liner materials. Firstly, as shown in Figs. 7 and 8, the coefficient of friction and energy dissipation of the 

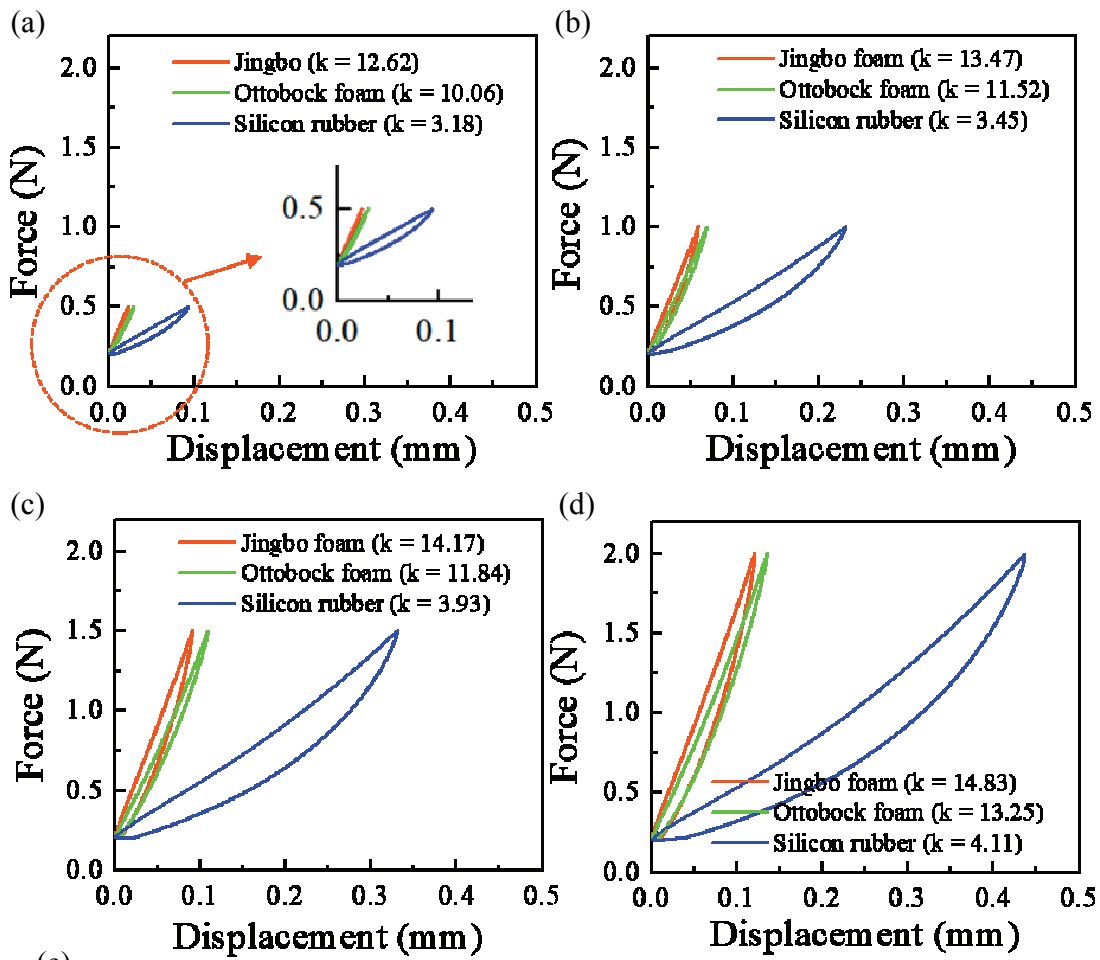

(e)
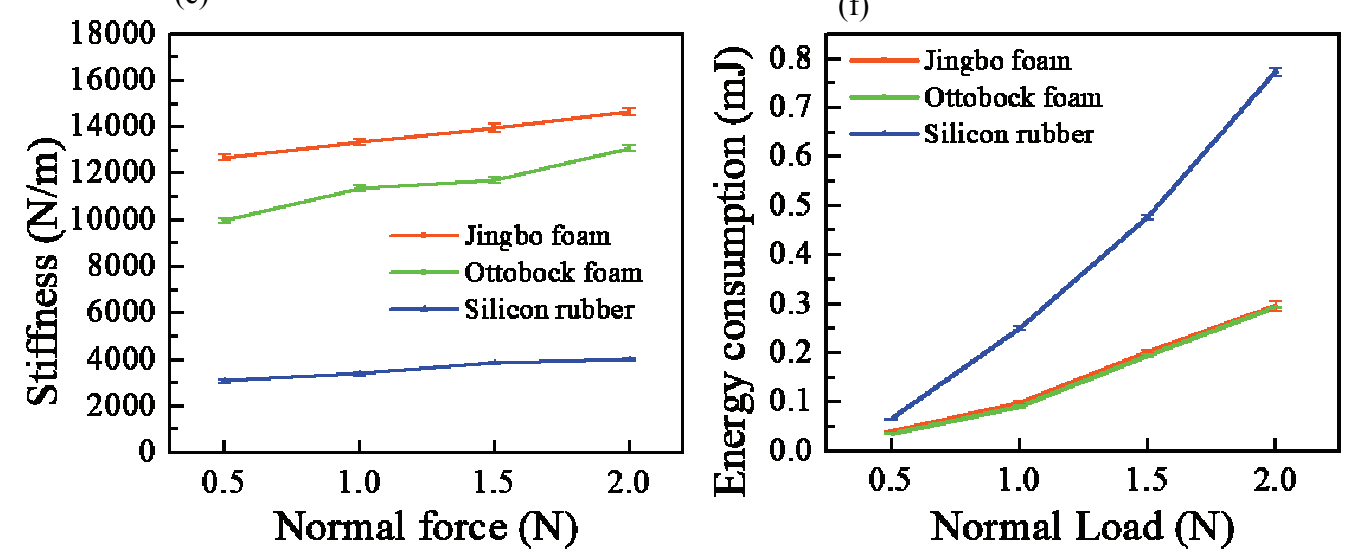

Fig. 10. Typical loading-unloading curves of prosthetic liner materials under different compressive loads, the stiffness and the energy consumption of different prosthetic liner materials:

(a) typical loading-unloading curve under $0.5 \mathrm{~N}$; (b) typical loading-unloading curve under $1.0 \mathrm{~N}$;

(c) typical loading-unloading curve under $1.5 \mathrm{~N}$; (d) typical loading-unloading curve under $2.0 \mathrm{~N}$;

(e) the stiffness under different compressive loads; (f) the energy consumption in one loading-unloading cycle under different compressive loads

3D-printed materials are close to each other, and they are greater than that of the acrylic socket material. The results have a positive correlation to the surface roughnesses listed in Table 1 and the surface roughnesses of the 3D-printed materials are almost similar, and are greater than that of the acrylic socket material. It causes higher coefficient of friction and energy dissipation when they rubbing against the same liner under the same normal load. Secondly, for the different prosthetic liner materials, the surface roughnesses of the Ottobock foam liner and the Jingbo foam liner are almost the same (Table 1). So, the coefficient of friction and energy consumption between them are not significantly different $(P>0.05)$.

Regarding the composition of reinforced materials in polymer materials, Zhao et al. [24] found that under dry sliding condition, the carbon fiber reinforced polyimide composite exhibits lower coefficient of friction than that of the glass fiber reinforced polyimide composite. In this study, similar result is also evident in Fig. 7b. In addition, Wang et al. [20] drew a conclusion that carbon fiber reinforced PEEK (polyether ether ketone) composites offer a far superior wear resistance over UHMWPE (ultra high mo- 
lecular weight polyethylene) against either metal or ceramic head in a conforming ball-in-socket contact situation such as in the hip joint. Therefore, type of the reinforcement fiber influences the friction behavior of the 3D-printed fiber reinforced polymer socket material.

For the liner materials, the coefficient of friction and energy consumption of the silicon rubber liner are considerably lower than that of the two foam liners $(P<0.05)$, indicating that they do not depend on the surface roughness, but on the stiffness of the materials. Stiffness is an important mechanical property of the liner materials [16]. As shown in Fig. 10e, the stiffness of the silicone rubber is lower than that of the two foam materials $(P<0.05)$. It means that the silicon rubber liner is easier to be deformed during the friction process. In addition, during the friction period, the partial deformation of the liner material reduced the coefficient of friction of the interface. As a result, the coefficient of friction of silicone rubber is lower than that of the two foam materials (Fig. 7). Moreover, as shown in Fig. 10f, the energy consumption of the silicone rubber is the largest during compression deformation process, which means its energy consumption is the largest. It can be seen its frictional energy dissipation and temperature increase are the smallest among the three liner materials (Figs. 8 and 9). Furthermore, since the energy consumption of the liner materials increased with the compressive load, the temperature increase under normal load of $8.0 \mathrm{~N}$ is dramatically higher than that under normal load of $0.8 \mathrm{~N}$.

As can be seen from Figs. 5 and 6, the scratch shows on the surface of the two kinds of foam liners after friction, and the surface roughnesses increased. In comparison, the scratch of the Jingbo foam liner is severer, and the increase value of surface roughness is greater than that of the Ottobock foam liner. This could be caused by the wear resistance of the foam liner. Another possible reason is that the surface roughness of the Ottobock foam liner is slightly less than that of the Jingbo foam liner. Moreover, it is noted that the increase of surface roughness is comparatively smaller after friction with the acrylic materials, which indicates that the comparatively smooth surface of the prosthetic socket can prevent scratch on the surface of the foam liner.

Since the foam liner is fixed on the prosthetic socket by friction, it is expected that the friction at the interface be greater to prevent the sliding at the prosthetic socket/liner interface and increase the stability during walking period [4], [5]. Therefore, it is feasible to increase the friction force rationally through the design of surface structure [6]. For the silicone rubber liner, it is fixed in the prosthetic socket by a lock screw. The appropriate friction between the socket and liner is important for the patient to put the residual limb with liner into the ideal position, therefore, the fabric layer on the silicone rubber liner can reduce the coefficient of friction at the contact interface, facilitating the amputee to put on and take off the prosthesis, and also protect the liner from abrasion to increase the durability. In addition, from the perspective of friction energy consumption at the interface, the silicone rubber liner with low stiffness and high energy consumption can reduce the friction energy dissipation at the contact interface, protecting the residual limb skin and improving the wear comfort. Besides, the amputees have a general preference for a silicon rubber liner, because it can offer skin protection and diminish friction between the socket and the surface of residual limb [1].

\section{Conclusions}

In conclusion, the friction behavior at the interface between the prosthetic socket and liner was investigated under the simulated friction conditions. Through a series of tests and analyses, the following conclusions can be drawn:

(1) The surface roughness has a significant impact on coefficient of friction and energy dissipation between the prosthetic socket and liner materials. Compared to the smooth handmade acrylic socket material, the three kinds of 3D-printed materials with higher surface roughness have greater coefficient of friction and energy dissipation at the contact interface.

(2) The stiffness and energy consumption of the prosthetic liner materials play an important role in the friction characteristics. The low stiffness and high energy consumption of the silicone rubber liner can reduce the coefficient of friction, energy dissipation and temperature rise at the contact interface.

(3) As coefficient of friction at the interface between prosthetic socket and liner has an impact on the difficulty level in prosthetic socket wearing and the adaptability of the prosthesis, the appropriate coefficient of friction at the surface between prosthetic socket and liner is essential.

(4) Type of the reinforcement fiber influences the friction behavior of the 3D-printed fiber-reinforced nylon socket. 


\section{Declaration of competing interest}

The authors declare that they have no known competing financial interests or personal relationships that could have appeared to influence the work reported in this paper.

\section{Acknowledgements}

This work was supported by National Natural Science Foundation of China (No. 51675447 and No. 51175440) and China Assistive Devices and Technology Centre (No. CJFJRRB10-2018).

\section{References}

[1] BAARS E.C.T., GEERTZEN J.H., Literature review of the possible advantages of silicon liner socket use in transtibial prostheses, Prosthetics and Orthotics International, 2005, 29 (1), 27-37.

[2] Bourell D., Montgomery J.T., Vaughan M.R., CRAWFORD R.H., Design of an actively actuated prosthetic socket, Rapid Prototyping Journal, 2010, 16 (3), 194-201.

[3] Cagle J.C., Reinhall P.G., Allyn K.J., Mclean J., HinRichs P., HafNer B.J., SANDERS J.E., A finite element model to assess transtibial prosthetic sockets with elastomeric liners, Medical and Biological Engineering and Computing, 2018, 56, 1227-1240.

[4] Cavaco A., Durães L., Pais S., Ramalho A., Friction of prosthetic interfaces used by transtibial amputees, Biotribology, 2016, 6, 36-41.

[5] EMrich R., Slater K., Comparative analysis of below-knee prosthetic socket liner materials, Journal of Medical Engineering and Technology, 1998, 22 (2), 94-98.

[6] Feng Q.P., Li W., LiU X.D., Ji W., Zhou Z.R., Investigation of reciprocating friction characteristics between different bionic surfaces of prosthesis materials and skin, Biosurface and Biotribology, 2019, 5 (2), 57-66.

[7] Florence M.M., ANDREW J.C., JoshuA W.S., Dickinson A.S., Predictive control for an active prosthetic socket informed by FEA-based tissue damage risk estimation, 41st Annual International Conference of the IEEE Engineering in Medicine and Biology Society (EMBC), 2019.

[8] JiA X.H., Zhang M., Li X.B., LeE W.C.C., A quasi-dynamic nonlinear finite element model to investigate prosthetic interface stresses during walking for trans-tibial amputees, Clinical Biomechanics, 2005, 20, 630-635.

[9] KeJlaA G.H., Consumer concerns and the functional value of prostheses to upper limb amputees, Prosthetics and Orthotics International, 2009, 17 (3), 157-163.

[10] Kong M., Li W., Li H.L., LiU X.D., Zhou Z.R., The skin frictional properties of 4 kinds of commonly used prosthetic materials, Journal of Biomedical Engineering, 2008, 25 (5), $1107-1111,1125$

[11] Lee W.C.C., Zhang M., JiA X.H., Cheunga J.T.M., Finite element modeling of the contact interface between transtibial residual limb and prosthetic socket, Medical Engineering and Physics, 2004, 26 (8), 655-662.

[12] Li W., Kong M., LiU X.D., Zhou Z.R., Tribological behavior of scar skin and prosthetic skin in vivo, Tribology International, 2008, 41 (7), 640-647.

[13] Li W., LiU X.D., CAi Z.B., Zheng J., Zhou Z.R., Effect of prosthetic socks on the frictional properties of residual limb skin, Wear, 2011, 271 (11-12), 2804-2811.

[14] Li W., QU S.X., ZHOU Z.R., Reciprocating sliding behaviour of human skinin vivoat lower number of cycles, Tribology Letters, 2006, 23 (2), 165-170.

[15] Li W., Shi L., Deng H.Y., Zhou Z.R., Investigation on friction trauma of small intestine in vivo under reciprocal sliding conditions, Tribology Letters, 2014, 55 (2), 261-270.

[16] Lin C.C., Chang C.H., Wu C.L., Chung K.C., Liao I.C., Effects of liner stiffness for trans-tibial prosthesis: a finite element contact model, 2004, 26 (1), 1-9.

[17] Millstein S.G., Heger H., Hunter G.A., Prosthetic use in adult upper limb amputees: A comparison of the body powered and electrically powered prostheses, Prosthetics and Orthotics International, 2009, 10 (1), 27-34.

[18] Omasta M., David P., NÁvrat T., RosickÝ J., Finite element analysis for the evaluation of the structural behaviour, of a prosthesis for trans-tibial amputees, Medical Engineering and Physics, 2012, 34 (1), 38-45.

[19] Pohjolainen T., Alaranta H., KärkKäInEn M., Prosthetic use and functional and social outcome following major lower limb amputation, Prosthetics and Orthotics International, 1990, 14 (2), 75-79.

[20] Wang A., Lin R., Stark C., Dumbleton J.H., Suitability and limitations of carbon fiber reinforced PEEK composites as bearing surfaces for total joint replacements, Wear, 1999, 225-229 (4), 724-727.

[21] Zhang M., Lord M., Turner-Smith A.R., Roberts V.C., Development of a non-linear finite element modelling of the below-knee prosthetic socket interface, Medical Engineering \& Physics, 1995, 17 (8), 559-566.

[22] ZhANG M., RoBerTs C., Comparison of computational analysis with clinical measurement of stresses on below-knee residual limb in a prosthetic socket, Medical Engineering and Physics, 2000, 22 (9), 607-612.

[23] Zhang M., Turner-Smith A.R., Roberts V.C., TANner A., Frictional action at lower limb/prosthetic socket interface, Medical Engineering \& Physics, 1996, 18 (3), 207-214.

[24] Zhao G., Hussainova I., Antonov M., Wang Q.H., WANG T.M., Friction and wear of fiber reinforced polyimide composites, Wear, 2013, 301 (1-2), 122-129.

[25] ZHENG S.X., Zhao W., Lu B., Three dimensional finite $d y$ namic analysis of the residual limb and prosthetic socket, Journal of Xi'an Jiaotong University, 2006, 40 (7), 807. 